\title{
AMPK-mTOR-ULK1-mediated autophagy protects carbon tetrachloride-induced acute hepatic failure by inhibiting p21 in rats
}

\author{
Qiwen Wang1-6, Weixia Liu ${ }^{1-6}$, Gaopeng Liu' ${ }^{1-6}$, Pan $\mathrm{Li}^{1-6}$, Xueqiang Guo ${ }^{1-6}$, and \\ Chunyan Zhang1-6* \\ ${ }^{1}$ State Key Laboratory Cell Differentiation and Regulation, Henan Normal University, \#46 East of Construction Road, Xinxiang, \\ 453007 Henan, China \\ ${ }^{2}$ Henan International Joint Laboratory of Pulmonary Fibrosis, Henan Normal University, \#46 East of Construction Road, Xinxiang, \\ 453007 Henan, China \\ ${ }^{3}$ Henan Center for Outstanding Overseas Scientists of Pulmonary Fibrosis, Henan Normal University, \#46 East of Construction \\ Road, Xinxiang, 453007 Henan, China \\ ${ }^{4}$ College of Life Science, Henan Normal University, \#46 East of Construction Road, Xinxiang, 453007 Henan, China \\ 5 Institute of Biomedical Science, Henan Normal University, \#46 East of Construction Road, Xinxiang, 453007 Henan, China \\ ${ }^{6}$ Overseas Expertise Introduction Center for Discipline Innovation of Pulmonary Fibrosis (111 Project), Henan Normal University, \\ \#46 East of Construction Road, Xinxiang, 453007 Henan, China
}

\begin{abstract}
Autophagy is a lysosomal-dependent degradation pathway in eukaryotic cells. Recent studies have reported that autophagy can facilitate the activation of hepatic stellate cells ( $\mathrm{HSCs})$ and fibrogenesis of the liver during long-term carbon tetrachloride $\left(\mathrm{CCl}_{4}\right)$ exposure. However, little is known about the role of autophagy in $\mathrm{CCl}_{4}$-induced acute hepatic failure (AHF). This study aimed to identify whether modulation of autophagy can affect $\mathrm{CCl}_{4}$-induced $\mathrm{AHF}$ and evaluate the upstream signaling pathways mediated by $\mathrm{CCl}_{4}-$ induced autophagy in rats. The accumulation of specific punctate distribution of endogenous LC3-II, increased expression of LC3-II, Atg5, and Atg7 genes/proteins, and decreased expression of p62 gene were observed after acute liver injury was induced by $\mathrm{CCl}_{4}$ in rats, indicating that $\mathrm{CCl}_{4}$ resulted in a high level of autophagy. Moreover, loss of autophagic function by using chloroquine (CQ, an autophagic inhibitor) aggravated liver function, leading to increased expression of p21 (a cyclin-dependent kinase inhibitor) in $\mathrm{CCl}_{4^{-}}$ treated rats. Furthermore, the AMPK-mTORC1-ULK1 axis was found to serve a function in $\mathrm{CCl}_{4}$-induced autophagy. These results reveal that AMPK-mTORC1-ULK1 signaling-induced autophagy has a protective role in $\mathrm{CCl}_{4}$-induced hepatotoxicity by inhibiting the p21 pathway. This study suggests a useful strategy aimed at ameliorating $\mathrm{CCl}_{4}$-induced acute hepatotoxicity by autophagy. (DOI: 10.1293/tox.2020-0022; J Toxicol Pathol 2021; 34: 73-82)
\end{abstract}

Key words: carbon tetrachloride $\left(\mathrm{CCl}_{4}\right)$, autophagy, p21, AMPK-mTOR-ULK1 axis, rat

\section{Introduction}

Acute hepatic failure (AHF) is a liver function abnormality that results from chemicals, drugs, viruses, and toxins, potentially eventually leading to hepatic encephalopathy and cirrhosis. Among them, AHF induced by chemicals, such as carbon tetrachloride $\left(\mathrm{CCl}_{4}\right)$, is ideal for in vivo toxicity research. $\mathrm{CCl}_{4}$ has been reported to induce hepatotoxicity by activating the phase I cytochrome P450 system, which leads to the formation of free radicals and a series

Received: 8 April 2020, Accepted: 26 October 2020 Published online in J-STAGE: 5 January 2021

*Corresponding author: C Zhang (e-mail: zcy1119sdc@163.com) (C)2021 The Japanese Society of Toxicologic Pathology

This is an open-access article distributed under the terms of the Creative Commons Attribution Non-Commercial No Derivatives (C) $($ ) (by-nc-nd) License. (CC-BY-NC-ND 4.0: https:// (c) ${ }_{\text {BY }}$ NC creativecommons.org/licenses/by-nc-nd/4.0/). of oxygen active substances, further undergoing peroxidation with membrane lipids and destroying the integrity of the cell membrane structure of hepatocytes ${ }^{1,2}$. Moreover, the metabolites of $\mathrm{CCl}_{4}$ can also irreversibly and covalently bind to various intracellular macromolecules, leading to the reduction of glutathione and loss of mitochondrial function in the liver ${ }^{3,4}$. Prior studies have discovered that early stress in liver injury may be the result of increased energy requirements per unit of liver mass, and the remnant liver tissue retains liver-specific functions, such as gluconeogenesis, urea synthesis, and continuous production of proteins, nucleic acids as well as other cellular components ${ }^{5}$. However, the mechanisms underlying the recovery of liver function, including the role of metabolism and recombination of cellular components after AHF induced by $\mathrm{CCl}_{4}$, have not been fully elucidated.

Autophagy, a conserved evolutionary lysosomal process for the degradation and recycling of misfolded proteins, organelles, lipid droplets and pathogens, is widely 
considered a cytoprotective mechanism to maintain cellular homeostasis and prevent organism damage under adverse stress conditions ${ }^{6,7}$. For example, a recent report has confirmed that autophagy protects against cadmium-induced cytotoxicity in primary rat proximal tubular cells ${ }^{8}$. Accumulating evidence has also shown that autophagy plays an important role in maintaining liver homeostasis. It has been demonstrated that basal autophagy degrades $30 \%$ of liver proteins in wild-type mice after $24 \mathrm{~h}$ of starvation, which becomes insignificant in conditional knockout mice of Atg79. Suppression of basal autophagy could lead to hepatomegaly, which is followed by inflammation, hepatitis and tumorigenesis ${ }^{10}$. Moreover, aberrant expression of autophagy-related proteins was also found in certain hepatic pathological processes, such as ischemia-reperfusion, fatty liver, viral hepatitis and hepatic tumor ${ }^{11,12}$, indicating that autophagy plays an important role in normal and diseased livers. Our previous study demonstrated that Reg-mediated signaling pathways may account for the activation of inflammation and cell proliferation, along with the attenuation of apoptosis and cell death during the occurrence of AHF13. The aim of the present study was to establish the role of autophagy in $\mathrm{CCl}_{4}$-induced AHF in rats.

\section{Materials and Methods}

\section{Experimental animals}

Healthy adult male SD rats, which weighed 190-230 g supplied by the Experimental Animal Center of Zhengzhou University (Zhengzhou, China), were housed in a standard controlled room $\left(22 \pm 1^{\circ} \mathrm{C}\right)$ with relative humidity of $60 \pm$ $10 \%$ with a $12 \mathrm{~h}$ light-dark cycle where light periods were from 6:00-18:00. Rats were raised according to clean grade standards and did not have disease or other adverse symptoms. The Chinese Animal Protection Law was strictly adhered to during the experiment.

\section{Preparation of AHF model}

A total of 40 male rats were housed in a standard temperature room $\left(22 \pm 1^{\circ} \mathrm{C}\right)$ with a $12: 12 \mathrm{~h}$ light-dark cycle. $\mathrm{CCl}_{4}$ was diluted with sesame oil $(2: 3, \mathrm{v} / \mathrm{v})$ under sterile conditions. The rats in the AHF group were fed with diluted $\mathrm{CCl}_{4}$ at a dose of $4 \mathrm{~mL} / \mathrm{kg}$ body weight ${ }^{13}$. Rats in the chloroquine (CQ) treatment group received intraperitoneal injection of CQ (30 mg/kg, Sigma-Aldrich, St. Louis, MO, USA) once daily for 7 days before $\mathrm{CCl}_{4}$ exposures. After treatment, the right liver lobes and posterior vena cava were taken for subsequent experiments at each of the following time points: $0,3,6,12$ and $24 \mathrm{~h}$.

\section{Western blotting}

Liver tissues were cut into pieces and lysed with RIPA buffer (Beijing Solarbio Science \& Technology Co., Ltd., Beijing, China) supplemented with protease inhibitors (Beijing Applygen Co., Ltd., Beijing, China) on ice for $30 \mathrm{~min}$ and centrifuged at $12,000 \times \mathrm{g}$ for $20 \mathrm{~min}$ at $4^{\circ} \mathrm{C}$. The protein concentration was determined by the BCA method accord- ing to the manufacturer's instructions (Beyotime Institute of Biotechnology, Haimen, China). Total proteins $(20 \mu \mathrm{g})$ were separated via $12-15 \%$ SDS polyacrylamide gel electrophoresis (PAGE) and transferred to nitrocellulose membranes (Beyotime Institute of Biotechnology). After blocking at room temperature for $2 \mathrm{~h}$ with $5 \%$ non-fat milk in TBS with $0.1 \%$ Tween 20 , the membranes were incubated overnight at $4^{\circ} \mathrm{C}$ with antibodies against BECN1 (cat. no. 3495), Atg5 (cat. no. 12994), Atg7 (cat. no. 8558), and Akt (cat. no. 4691), p-Akt (Thr308) (cat. no. 13038), Raptor (cat. no. 2280), P-Raptor (Ser792) (cat. no. 2083), AMPKa (cat. no. 5832), P-AMPK $\alpha$ (Thr172) (cat. no. 2535), ULK1(cat. no. 8054), P-ULK1 (Ser555) (cat. no. 5869), $\beta$-actin (cat. no. 4970) and HRP-conjugated secondary antibodies (cat. no. 7074) at room temperature for $1.5 \mathrm{~h}$; all antibodies were purchased from Cell Signaling Technology (Danvers, MA, USA). Signals were visualized with Amersham ECL substrates, and the relative levels of protein in each group were normalized to $\beta$-actin.

\section{Quantitative RT-PCR ( $q R T-P C R)$ analysis}

Total RNA was extracted from frozen liver tissue using Trizol (Invitrogen Corporation, Carlsbad, CA, USA) according to the manufacturer's instructions. RNA purity was verified by spectrophotometry at $260 \mathrm{~nm}$ and $280 \mathrm{~nm}$ absorbance values (A260/280) using a NanoDrop. Qualified RNA $(2 \mu \mathrm{g})$ was used to synthesize the first strand of cDNA with the Reverse Transcription kit (Promega, Madison, Wl, USA). The cDNA was employed for PCR amplification with SYBR Green master mix. $\beta$-actin was used as an internal reference. Three independent experiments were performed for each group. The sequences of the primers are listed in Table 1 .

\section{Hematoxylin-eosin (H\&E) staining}

Histopathological changes in liver tissues with AHF were examined by H\&E staining according to our laboratory methods previously described ${ }^{13}$. Briefly, approximately $5 \times 5 \times 3-\mathrm{mm}$ cuboids from the right lobe of the liver were routinely fixed, washed, dehydrated, cleared and embedded in paraffin. Afterwards, liver sections $(4 \mu \mathrm{m})$ were stained with H\&E (Beyotime Institute of Biotechnology) according to the manufacturer's instructions. The liver sections were

Table 1. The Primer Sequences Used in the qRT-PCR

\begin{tabular}{ll}
\hline Gene & Primer sequences \\
\hline ATG5 & Fwd:5'-CCACTGGAAGAATGACAGATGAC-3' \\
& Rev:5'-ATGCAAGAAGATCGAACAGTAGG-3' \\
ATG7 & Fwd:5'-CCCAAAGACATCAAGGGCTATT-3' \\
& Rev:5'-GGCAGAAAACCAGTAGTAGAAG-3' \\
LC3B & Fwd:5'-GATTATAGAGCGATACAAGG-3' \\
& Rev:5'-CTCGTACATTCAGAGATGG-3' \\
P62 & Fwd:5'-TCTTTCCCAACCCCTT-3' \\
& Rev:5'-GCTCTCCCCACATTC-3' \\
AMPKa & Fwd:5'-AACAAGCCCACCCGATTCT-3' \\
& Rev:5'-CAGGGTTCTTCCTTCGCACA-3' \\
B-actin & Few:5'-ACATCCGTAAAGACTTATGCCAACA-3' \\
& Rev:5'-GTGCTAGGAGCCAGGGCAGTAATCT-3' \\
\hline
\end{tabular}


dehydrated with gradient ethanol, cleared in xylene and sealed with neutral gum, and then histological assessment was performed under a microscope.

\section{Oil red $O$ staining}

The histological visualization of fat cells and neutral fat was observed by Oil red O staining (Sigma-Aldrich) during $\mathrm{CCl}_{4}$ treatment with or without CQ. In brief, approximately $5 \times 5 \times 3-\mathrm{mm}$ cuboids from the right lobe of the liver were fixed with $4 \%$ paraformaldehyde, cryoprotected with $30 \%$ sucrose, and then embedded with OCT compound (Sakura Finetek, Torrance, CA, USA). Then, 5- $\mu \mathrm{m}$ frozen slices were stained with Oil Red $\mathrm{O}$ for $15 \mathrm{~min}$ at room temperature. Afterwards, the dye was discarded and counterstained with hematoxylin. After pouring out the dye solution and using water to wash it until blue, glycerin-gelatin was used as a seal and observed under a microscope.

\section{Immunofluorescence}

Immunofluorescence analysis was carried out on the OCT compound embedded in the liver cryosection. Sections were fixed with $4 \%$ paraformaldehyde (Sinopharm Chemical Reagent Co., Ltd, Shanghai, China) at $4{ }^{\circ} \mathrm{C}$ for 30 min and then permeabilized with $1 \%$ Triton X-100 for 20 min. After three washes with PBS, the slices were blocked with 5\% BSA blocking solution and incubated with LC3B (cat. no. 3868) and p21 (cat. no. 2947) antibodies overnight at $4{ }^{\circ} \mathrm{C}$; these antibodies were purchased from Cell Signaling Technology (Danvers, MA, USA). The cells were then washed in blocking solution and stained with FITC-conjugated anti-rabbit IgG (Boster Biological Technology, Wuhan, China) for $1 \mathrm{~h}$. Cell nuclei were stained with honchest 33258 dye solution (Beyotime Institute of Biotechnology). Next, samples were examined under a fluorescence microscope (Nikon D-FL-E, Tokyo, Japan). Quantitation of 300 cells containing fluorescein isothiocyanate FITC-LC3 and FITC-p21 were analyzed by Image J (Bio-Rad Laboratories, Inc., Hercules, CA, USA).

\section{Detection of serum enzyme activity}

Serum was separated from the posterior vena cava. Serum aminotransferase levels were analyzed at Xinxiang Medical University.

\section{Statistical analysis}

All data are expressed as mean \pm standard deviation. The differences between groups were analyzed by one-way analysis of variance (ANOVA) with the Statistical Package for the Social Sciences (SPSS), v. 19.0 statistical software (IBM, Chicago, IL, USA). $P<0.05$ indicated significant differences while $P<0.01$ indicated extremely significant differences.

\section{Results}

Autophagy is activated after $\mathrm{CCl}_{4}$ treatment in rats

To examine whether autophagic activity is altered after $\mathrm{CCl}_{4}$ treatment in vivo, qRT-PCR analysis was used to detect the expression of autophagy-related genes. The results showed that expression of $L C 3 B, \operatorname{Atg} 5$ and $\operatorname{Atg} 7$ was significantly upregulated after $\mathrm{CCl}_{4}$ exposure, peaking at 6 $\mathrm{h}$, followed by a gradual decrease. However, p62, an autophagy adaptor molecule that brings ubiquitinated substrates to autophagosomes, exhibited the opposite expression trend (Fig. 1A-D). Autophagy-related proteins were also detected by Western blot, just as shown in Fig. 1E-H. An increase in BECN1, Atg5 and Atg7 was evident in a time-dependent manner after treatment with $\mathrm{CCl}_{4}$ compared with the control group. The distribution of endogenous LC3-II (LC3B) was analyzed by indirect immunofluorescence staining. As revealed in Fig. 1I, specific punctate distribution of endogenous LC3-II was increased in AHF samples. CQ, a pharmacologic inhibitor of autophagy, can decrease autophagosome-lysosome fusion, possibly resulting in a remarkable accumulation of autophagic vacuoles ${ }^{14}$. We also found more dots in the CQ- and $\mathrm{CCl}_{4}$-treated groups than in $\mathrm{CCl}_{4}$ alone $(P<0.05$, Fig. $1 \mathrm{~J})$. These results indicate that CQ treatment increases the accumulation of autophagosomes after $\mathrm{CCl}_{4}$ induced AHF.

\section{Inhibition of autophagy aggravates $\mathrm{CCl}_{4}$-induced hepatotoxicity}

To determine whether the loss of autophagic function affected $\mathrm{CCl}_{4}$-induced hepatotoxicity, hepatic histological changes were examined using the H\&E and Oil Red O staining assay. For the normal livers of rats, the liver lobules were clearly structured, cords were neatly arranged, and hepatocytes were uniformly distributed in the nucleus and cytoplasm. At-3-6 h of AHF, slight hydropic degeneration was observed. After treatment with $\mathrm{CCl}_{4}$ for 12 and $24 \mathrm{~h}$, bleeding and necrosis began to be observed (Fig. 2A), accompanied by the appearance of massive lipid droplets (Fig. 2B) in liver tissues. Furthermore, co-treatment with $\mathrm{CCl}_{4}$ and $\mathrm{CQ}$ further deteriorated hepatic injury (Fig. 2A and B). At the same time point, when rats were treated with $\mathrm{CCl}_{4}$ and $\mathrm{CQ}$, the levels of alanine aminotransferase (ALT) and aspartate aminotransferase (AST) in serum were significantly increased compared to those in the $\mathrm{CCl}_{4}$ treatment group $(P<0.01$, Fig. 2C). Collectively, these results indicate that $\mathrm{CCl}_{4}$-mediated autophagy has a hepatoprotective effect.

\section{Autophagy inhibition enhances the level of p21}

To identify whether $\mathrm{CCl}_{4}$-induced autophagy exerts an effect on the expression of p21 (a cyclin-dependent kinase inhibitor that is associated with senescence), indirect immunofluorescence analysis was conducted. As portrayed in Fig. 3, a greater number of endogenous p21 was observed in liver samples of AHF, and the increase was time-dependent, while faint fluorescence appeared in the control group $(P<0.01)$. Moreover, as expected, co-treatment with 


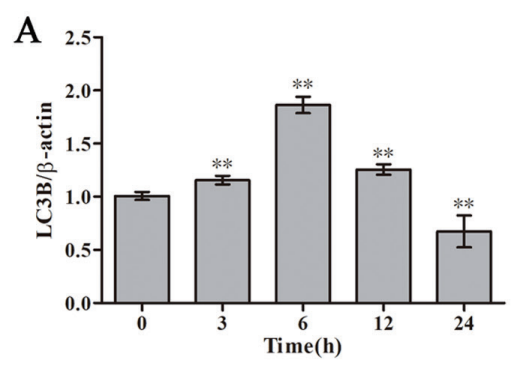

D

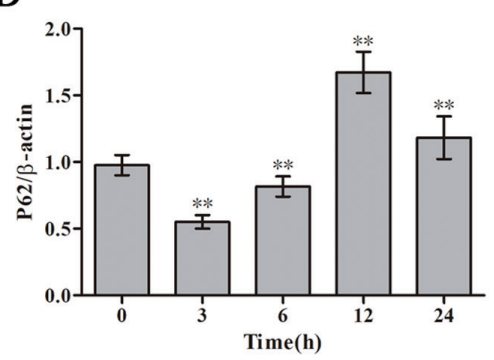

G
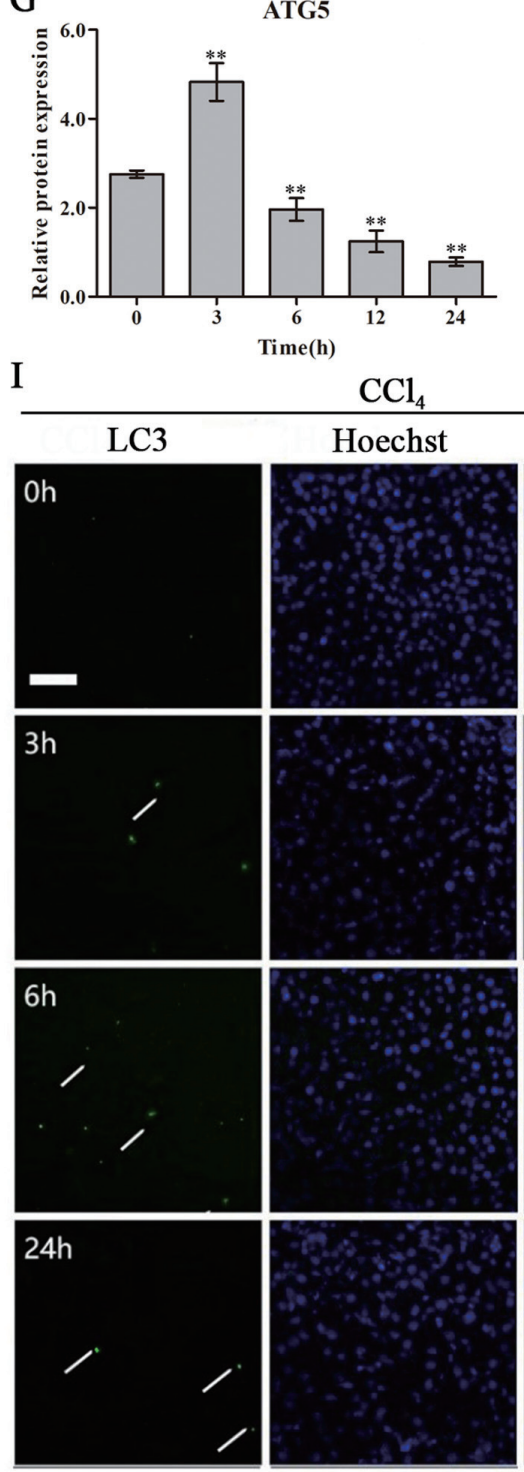

B

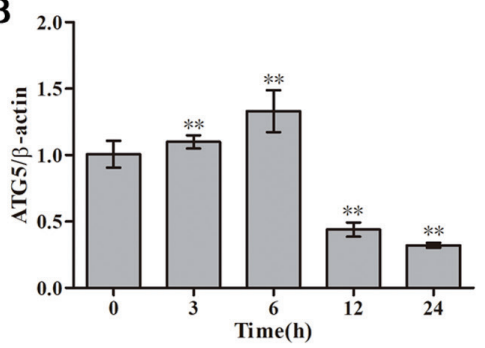

E

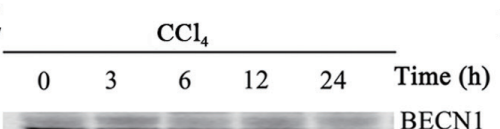

$-1-$ ATG5

$\longrightarrow-20$ ATG7

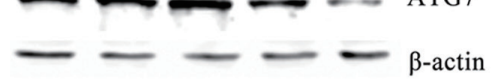

$\mathrm{H}$

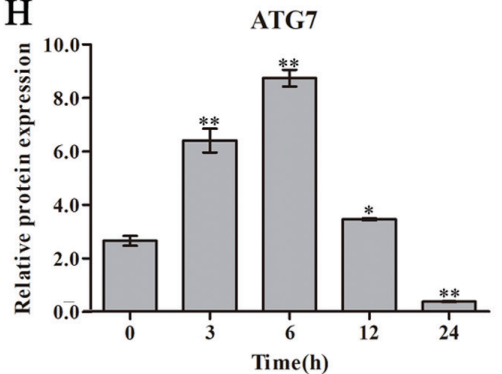

Merge
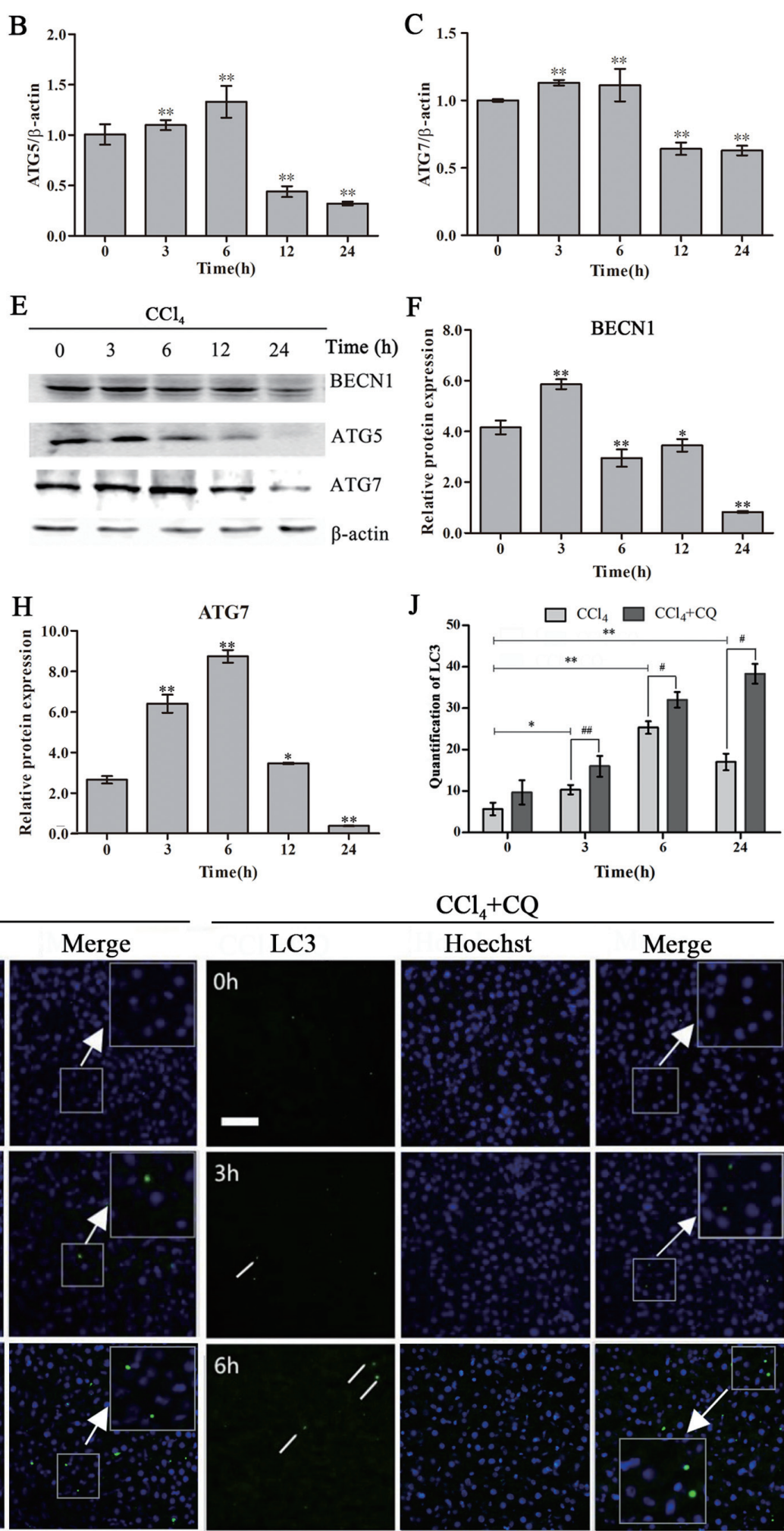

F
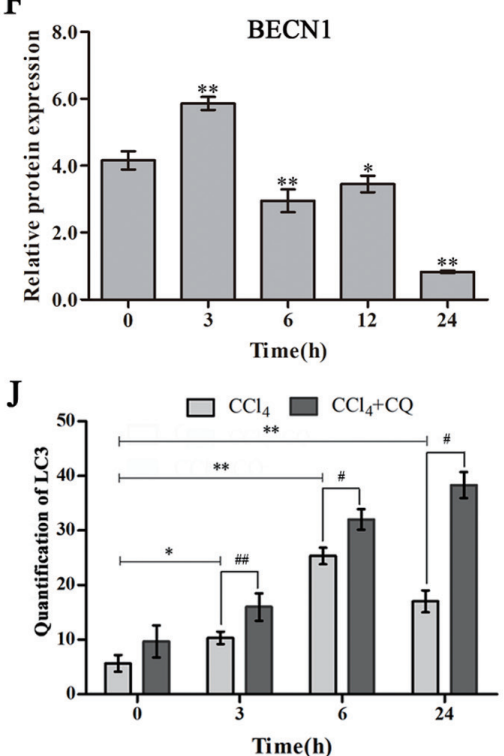

$\mathrm{CCl}_{4}+\mathrm{CQ}$

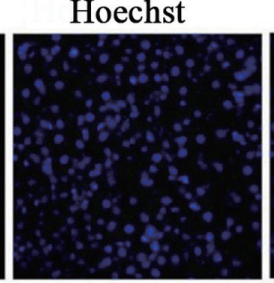

Merge
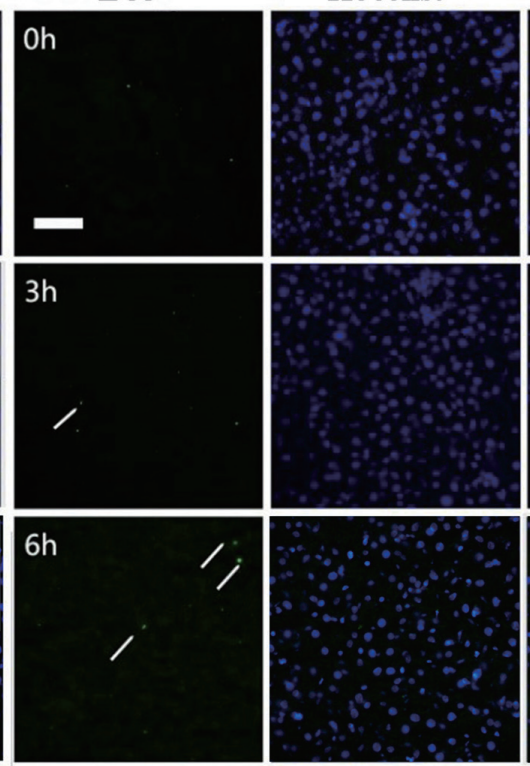

$24 \mathrm{~h}$
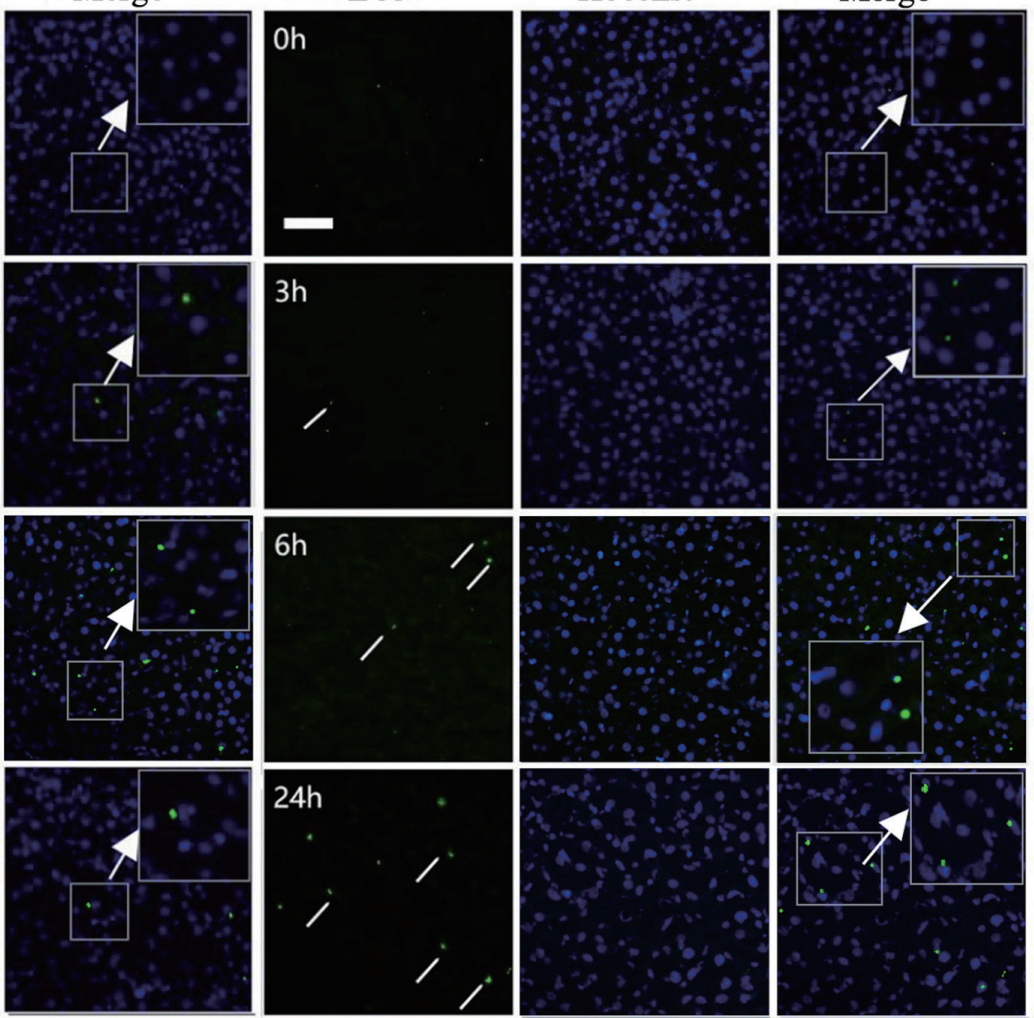

Fig. 1. 
$\mathrm{A}_{\mathrm{CCl}_{4}}$
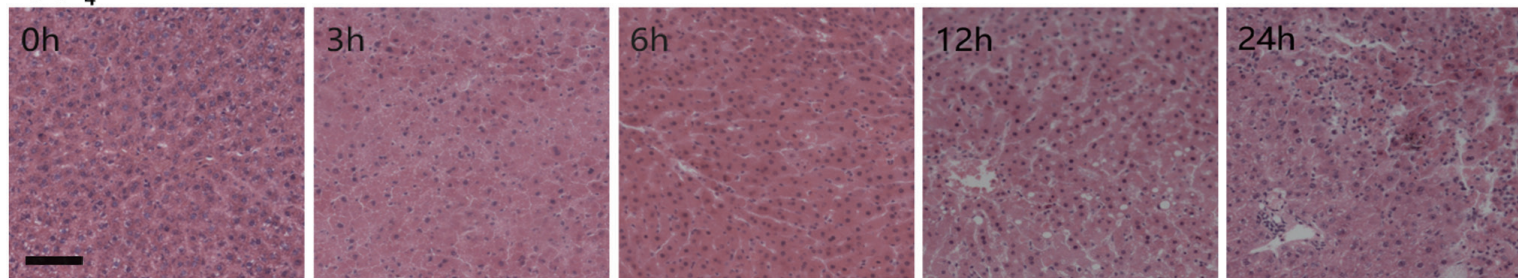

\section{$\mathrm{CCl}_{4}+\mathrm{CQ}$}
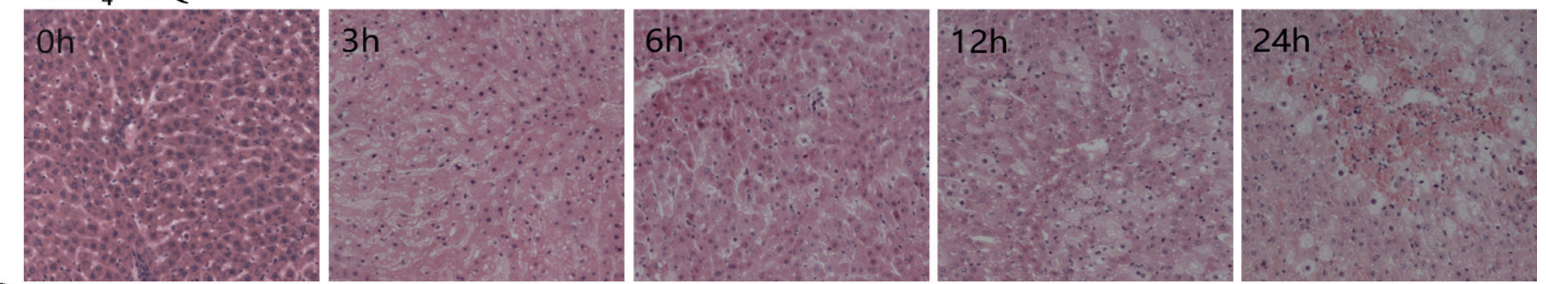

$\mathrm{B} \mathrm{CCl}_{4}$
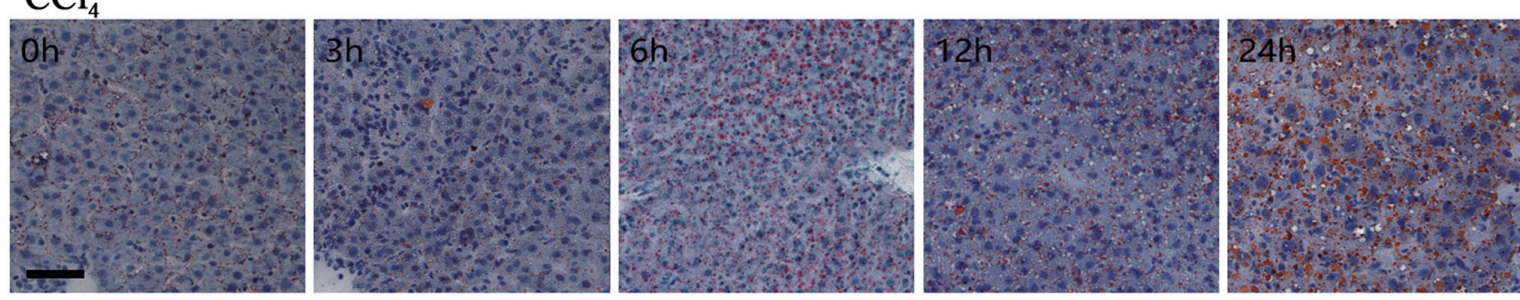

\section{$\mathrm{CCl}_{4}+\mathrm{CQ}$}
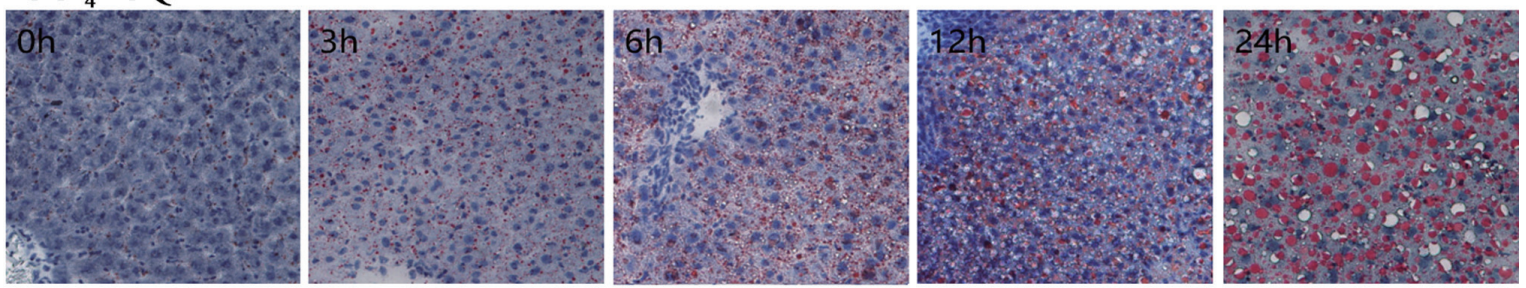

C
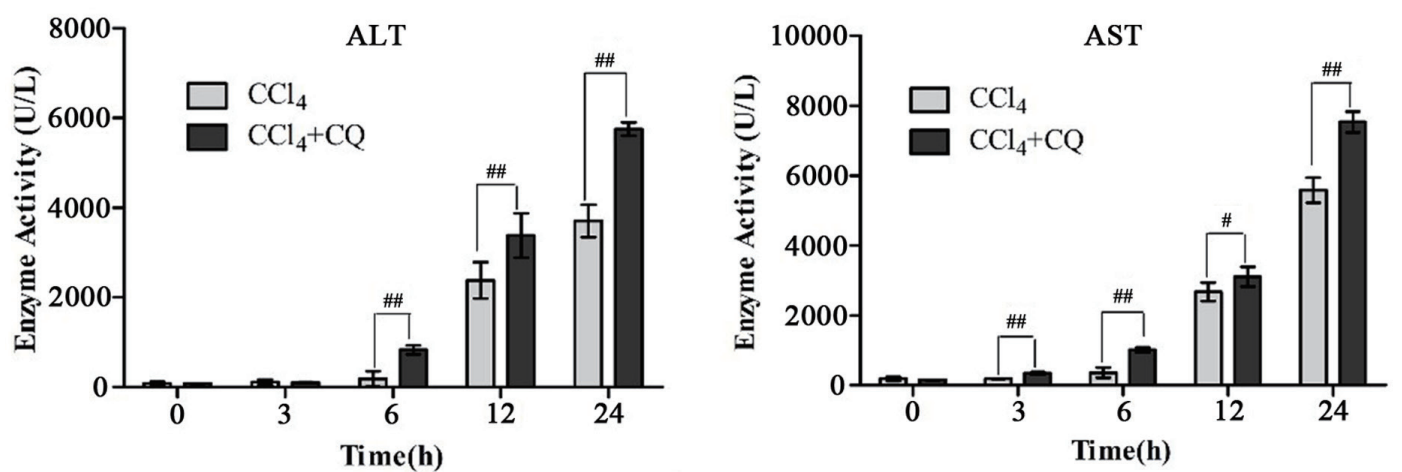

Fig. 2. Inhibition of autophagy aggravates carbon tetrachloride $\left(\mathrm{CCl}_{4}\right)$-induced hepatotoxicity. After $\mathrm{CCl}_{4}$ treatment in the presence or absence of chloroquine (CQ), hepatic histology (A), adipose conversion and triglyceride (B), and liver function (C) were analyzed by hematoxylin-eosin (H\&E), Oil red O and serum enzyme activity assays, respectively. Data are represented as the mean \pm SD $(n=4)$ and analyzed by one-way ANOVA with SPSS 19.0. $\# P<0.05, \# \# P<0.01$ compared with the respective $\mathrm{CCl}_{4}$ group. Scale bar: $100 \mu \mathrm{m}$.

Fig. 1. Autophagy is activated after carbon tetrachloride $\left(\mathrm{CCl}_{4}\right)$ treatment in rats. Expression of autophagy-related genes, $A T G 5, A T G 7, L C 3 B$ and $P 62$, was detected by qRT-PCR after $\mathrm{CCl}_{4}$ treatment for different durations, and relative mRNA levels were analyzed while $\beta$-actin was used as loading control (A-D). The expression of autophagy marker proteins, BECN1, Atg5 and Atg7, were detected by Western blotting (E) and quantitatively analyzed (F-H). $\beta$-actin was used as a loading control. LC3 puncta were observed by immunofluorescence after $\mathrm{CCl}_{4}$ treatment in the presence or absence of chloroquine (CQ). The oblique lines represent LC3 puncta (I). Histogram showing the number of punctate FITC-LC3 staining totaling $~ 300$ cells $(\mathrm{J})$. All data are represented as the mean \pm SD $(\mathrm{n}=4)$ and analyzed by oneway ANOVA with SPSS 19.0. ${ }^{*} P<0.05$, ${ }^{* *} P<0.01$ compared with the control group. $\# P<0.05$, \#\#P<0.01 compared to the respective $\mathrm{CCl}_{4}$ group. Scale bar: $100 \mu \mathrm{m}$. 


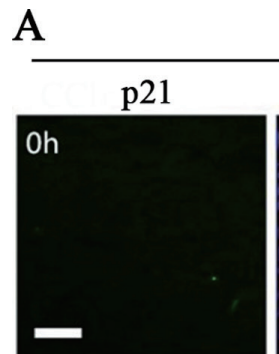

$\mathrm{CCl}_{4}$
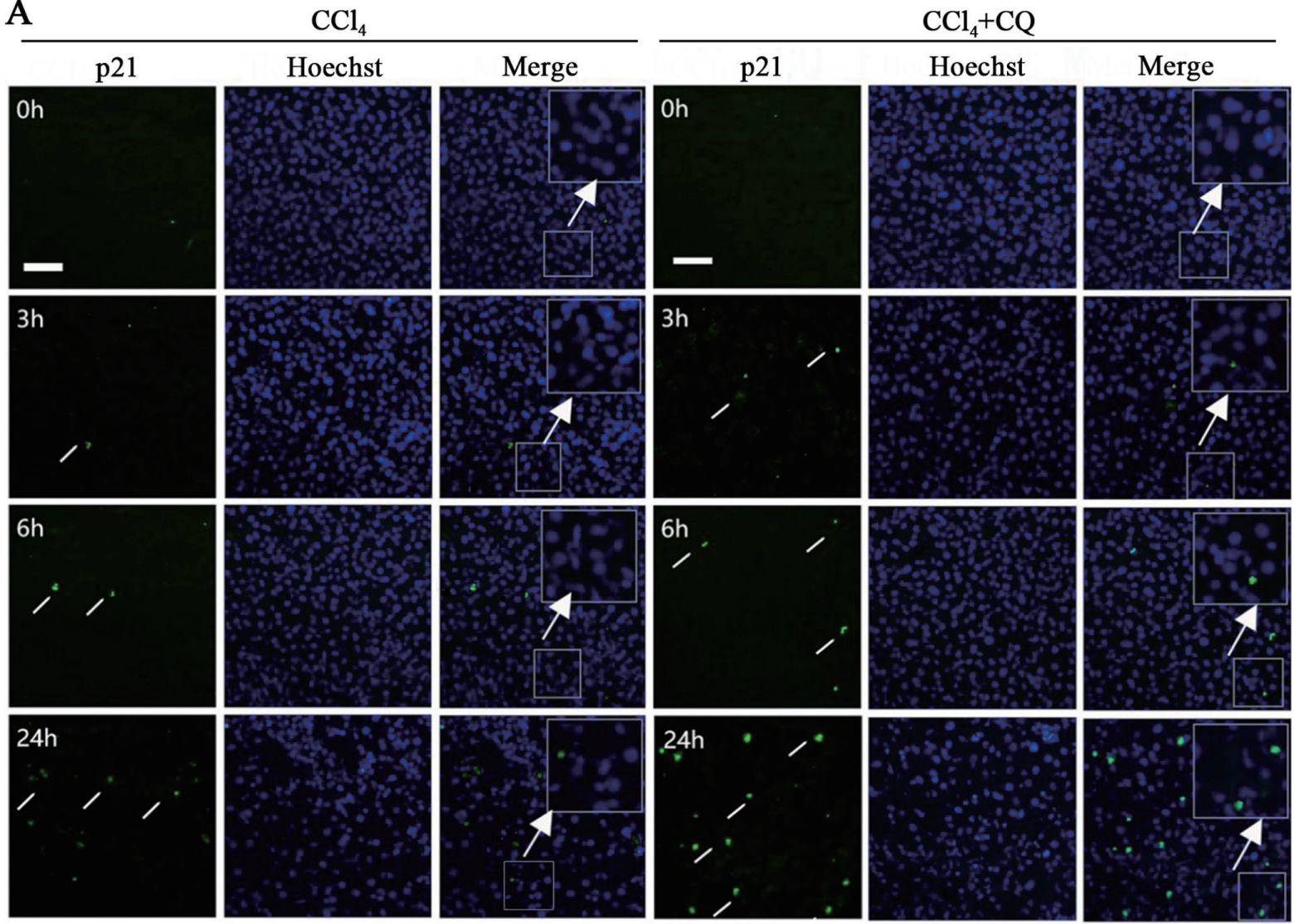

B

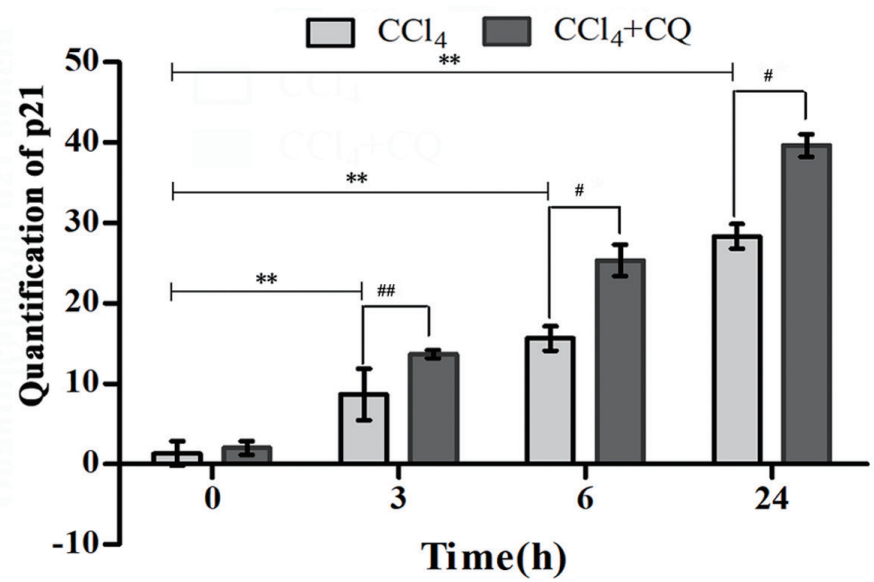

Fig. 3. Autophagy inhibition enhances the level of p21. Cyclin-dependent kinase inhibitor p21 was observed by immunofluorescence after carbon tetrachloride $\left(\mathrm{CCl}_{4}\right)$ treatment in the presence or absence of chloroquine (CQ) (A). The number of $\sim 300$ cells with punctate FITCp21 is displayed as a histogram (B). All data are represented as the mean \pm SD ( $\mathrm{n}=4)$ and analyzed by one-way ANOVA with SPSS 19.0 . $* * P<0.01$ compared with the control group. $\# P<0.05$, $\# \# P<0.01$, compared to the respective $\mathrm{CCl}_{4}$ group. The oblique lines represent the p21 puncta. Scale bar: $100 \mu \mathrm{m}$.

$\mathrm{CCl}_{4}$ and $\mathrm{CQ}$ further accelerated p21 protein accumulation compared with $\mathrm{CCl}_{4}$ treatment $(P<0.05)$, suggesting that autophagy may blunt $\mathrm{CCl}_{4}$-induced hepatotoxicity by inhibiting $\mathrm{p} 21$.
AMPK-mTORC1-ULK1 signaling contributes to autophagy activation in $\mathrm{CCl}_{4}$-induced $\mathrm{AHF}$

To investigate the signaling pathways involved in the induction of autophagy in $\mathrm{CCl}_{4}$-treated rat livers, the expression profiles of AMPK/mTOR signaling were evaluated by qRT-PCR and Western blotting. Investigators have confirmed that AMPK is a highly conserved energy regulator that is activated when there are even modest decreases 
A

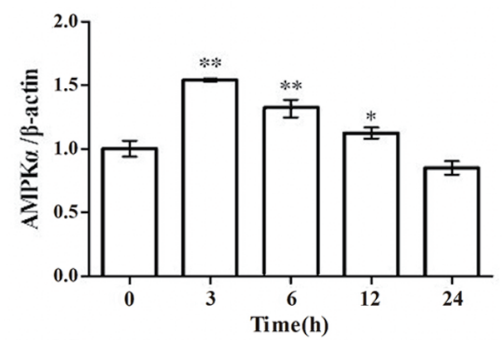

$\mathrm{C}$
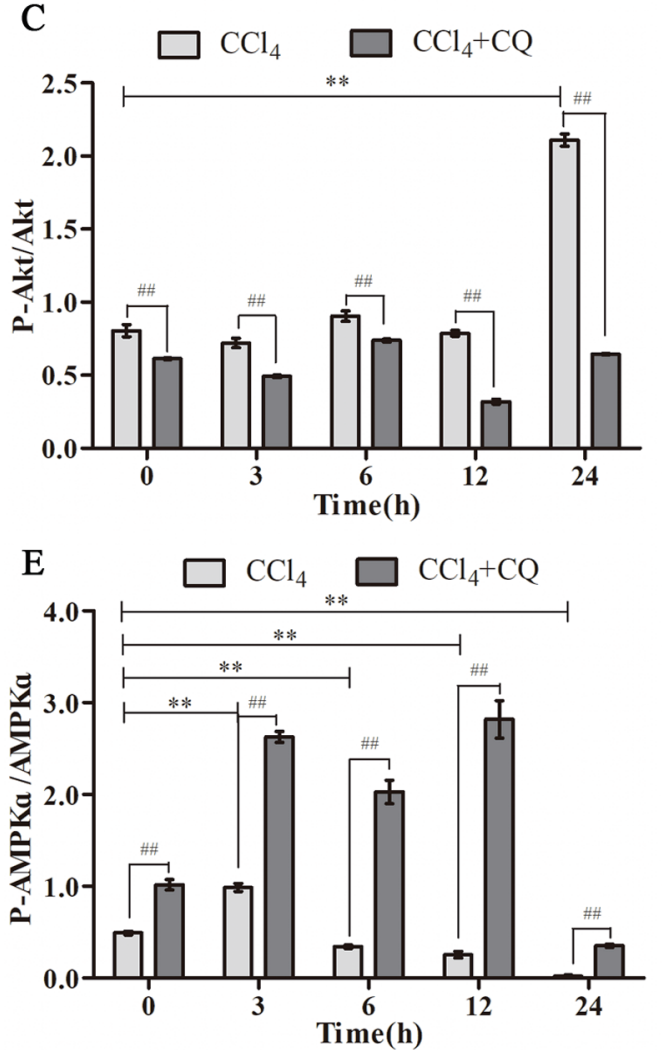

B

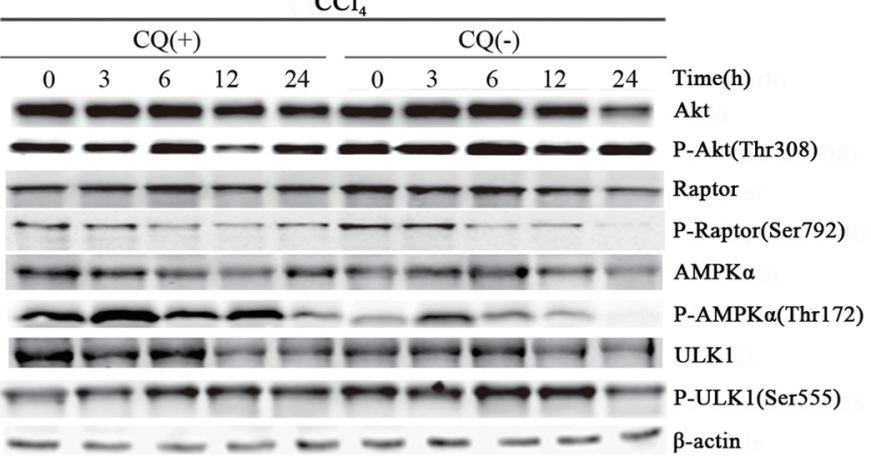

$\mathbf{D}$
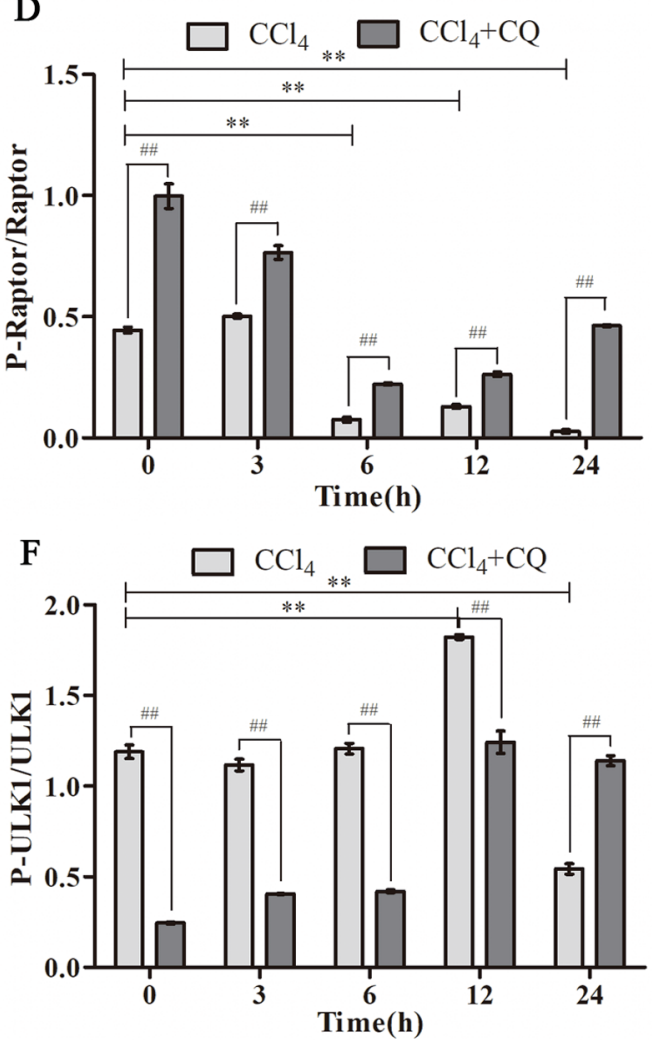

Fig. 4. AMPK/mTOR signaling pathway in carbon tetrachloride $\left(\mathrm{CCl}_{4}\right)$-induced acute hepatic failure (AHF). The expression of $\mathrm{AMPK} \alpha \mathrm{mRNA}$ was analyzed by qRT-PCR (A). AMPK/mTOR signaling proteins were detected (B) and quantitatively analyzed (C-F) after $\mathrm{CCl} \mathrm{L}_{4}$ treatment and $\mathrm{CCl}_{4}+$ chloroquine (CQ) treatment for different durations. $\beta$-Actin was used as a loading control. All data are represented as the mean $\pm \mathrm{SD}(\mathrm{n}=4)$ and analyzed by one-way ANOVA with SPSS 19.0. $* P<0.05, * * P<0.01$ compared with the control group. $\# \# P<0.01$, compared to the $\mathrm{CCl}_{4}$ group.

in ATP production ${ }^{15}$, so we first detected the expression of AMPK at the mRNA and protein levels. Unsurprisingly, $\mathrm{CCl}_{4}$ resulted in a significant upregulation of AMPK, and AMPK phosphorylation at threonine 172 (T172) in the $\alpha$-subunit is a key mechanism in the mediation of AMPK activation (Fig. 4A and B). Interestingly, P-ULK1 (Ser555) also showed a trend of first rising and then falling. Meanwhile, P-Raptor (Ser792) expression was decreased after treatment with $\mathrm{CCl}_{4}$ for 6,12 and $24 \mathrm{~h}$. However, there was no difference in P-Akt (Thr308) levels between the normal and AHF groups until $\mathrm{CCl}_{4}$ treatment for $24 \mathrm{~h}$ (Fig. 4B). We also found that, compared with the $\mathrm{CCl}_{4}$ treatment group, CQ co-treatment inhibited the phosphorylation of Akt and ULK1, but induced the phosphorylation of AMPK and Raptor $(P<0.01)$. These results suggest that the AMPKmTORC1-ULK1 signaling pathway could participate in autophagy induction after $\mathrm{CCl}_{4}$ treatment.

\section{Discussion}

Although recent studies highlight the involvement of autophagy in various animal models of liver injury, its mechanism still necessitates further exploration. In this study, the role of autophagy was investigated in $\mathrm{CCl}_{4}$-induced AHF. 
Our findings showed that $\mathrm{CCl}_{4}$ promotes autophagic activity in a time-dependent manner, which may relieve liver damage by inhibiting p21, and the AMPK-mTOR-ULK1 axis is involved in autophagy activation in $\mathrm{CCl}_{4}$-induced AHF.

The liver is an organ of great complexity with multiple functions. Recent work has shown that dysregulation of liver autophagy functions has an impact on pathologies of the liver, such as alcoholic and non-alcoholic fatty liver diseases as well as viral hepatitis ${ }^{11,12}$. However, very little is known about the role of autophagy in chemical-induced hepatotoxicity, especially $\mathrm{CCl}_{4}$. An earlier report demonstrated that autophagy in activated stellate cells is required for $\mathrm{CCl}_{4}-$ or thioacetamide-induced hepatic fibrogenesis - in mice, inhibition of autophagy by 3-methyladenine (3-MA) or small interfering RNAs against Atg5 or Atg7 effectively reduced HSC activation and fibrogenesis ${ }^{16}$. He et al. ${ }^{17}$ also observed that $\mathrm{CQ}$, another autophagy inhibitor, improves $\mathrm{CCl}_{4}$-induced liver fibrosis by downregulating the expression of profibrotic genes, such as $\alpha$-smooth muscle actin ( $\alpha$-SMA) and transforming growth factor (TGF- $\beta 1$ ). This indicates that autophagy participates in HSC activation and promotes the formation of liver fibrosis. However, there is accumulating evidence for protecting autophagy in response to $\mathrm{CCl}_{4}$. Pharmacological stimulation of autophagy by carbamazepine diminished hepatocellular death in patients with fibrinogen storage disease ${ }^{18}$. Interestingly, a recent study investigated activation of autophagy in $\mathrm{CCl}_{4}$-injured rat liver following transplantation with chorionic plate-derived mesenchymal stem cells (CP-MSCs). It was shown that necrosis and apoptosis were decreased; hypoxia-inducible factor- $1 \alpha$ (HIF-1 $\alpha$ ), autophagy and liver regeneration were significantly increased by CP-MSC transplantation. Moreover, the upregulation of HIF- $1 \alpha$ promotes the regeneration of damaged hepatic cells through autophagy ${ }^{19}$. Given the possibility of different sensitivity thresholds and liver compensation for $\mathrm{CCl}_{4}$, the exact nature of the relationship between autophagy and $\mathrm{CCl}_{4}$-induced liver injury is not well-elucidated. Our current findings suggest that autophagy appears to have a protective mechanism in AHF caused by $\mathrm{CCl}_{4}$. This observation is supported by how autophagy is activated after $\mathrm{CCl}_{4}$ treatment and CQ-mediated autophagy inhibition by increasing the levels of endogenous LC3-II (Fig. 1). In addition, intraperitoneal injection of CQ further exacerbated the hepatotoxicity induced by $\mathrm{CCl}_{4}$ (Fig. 2). These data are consistent with reports that autophagy promotes cell survival by removing damaged mitochondria and decreasing oxidative stress in cases of drug-induced hepatotoxicity, such as acetaminophen or efavirenz exposure ${ }^{20}$.

p21 is a cyclin-dependent kinase inhibitor (CDK) 1 or CDK-interacting protein 1 , which not only mediates growth arrest at specific stages in the cell cycle primarily by binding to and inhibiting CDK and PCNA ${ }^{21}$, but is also a major regulator of cellular senescence, a complex program involving multiple signaling pathways ${ }^{22}$. Previous reports have shown that hepatic mRNA and protein expression levels of p21 are higher in liver-specific Atg5 knockout mice than in control mice after partial hepatectomy, and upregulation of p21 was associated with hepatocyte senescence-associated $\beta$-galactosidase expression, which led to irreversible growth arrest and secretion of senescence-associated molecules ${ }^{5}$. Similarly, in $\mathrm{CCl}_{4}$-induced AHF, we found that $\mathrm{p} 21$ was significantly upregulated in a time-dependent manner, and CQ treatment further promoted the expression of $\mathrm{p} 21$ protein (Fig. 3). However, dihydroartemisinin-induced autophagy was not linked to senescence or cell death in HepG2.2.15 cells $^{23}$. In a recent report, Manu and colleagues ${ }^{24}$ also determined that $\mathrm{p} 21$ is an upstream regulator of autophagy through the transcriptional regulation of downstream effectors (BNIP3 and ULK1) in response to isoprenylcysteine carboxyl methyltransferase (an enzyme catalyzing the final step of protein prenylation) inhibition. In this study, we only investigated the level of p21 when autophagy was inhibited, but whether p21 depletion affects autophagy remains obscure. Therefore, further detailed research is required to clarify the relationship between autophagy and p21 in AHF induced by $\mathrm{CCl}_{4}$.

Various signaling pathways are involved in regulating autophagy, but the mechanisms underlying $\mathrm{CCl}_{4}$-induced autophagy continue to be unclear. Here, we focused our attention on the AMPK-mTORC1-ULK1 pathways. Our results indicated that AMPK-mTORC1-ULK1 signaling is activated in $\mathrm{CCl}_{4}$-induced hepatic injury (Fig. 4). Many studies have shown that AMPK activity is affected by AMP/ $\mathrm{ATP}^{15}$. When energy is scarce, AMP binds to AMPK $\gamma$ to initiate phosphorylation of AMPK $\alpha$ by LKB1, thereby activating AMPK. On the one hand, this takes place by opening up the catabolic pathways to produce ATP and turn off anabolism to reduce the consumption of ATP. On the other hand, AMPK activation inhibits the activity of mTORC1 by TSC1/2. As the central link in the development of autophagy, when mTORC1 activity is inhibited, it is separated from the Atg1/ULK complex to initiate autophagy ${ }^{25}$. It has been reported that thyroxin initiates autophagy via ROSAMPK-mTOR-ULK1 and participates in the regeneration and differentiation of myocytes ${ }^{26}$. Recently, investigators have pointed out that the AMPK/autophagy pathway is also closely related to hepatic disease. For example, activation of autophagy through the AMPK/mTOR pathway causes activation of rat hepatic stellate cells under hypoxic stress ${ }^{27}$ and ischemia-reperfusion injury ${ }^{28}$. Zeng et al. ${ }^{29}$ established that elimination of Kupffer cells can significantly improve hepatic inflammatory response and insulin tolerance in high-fat diet-fed mice, and its mechanism may be related to activation of AMPK/autophagy and NF- $\mathrm{B}$ signaling. For $\mathrm{CCl}_{4}$-induced $\mathrm{AHF}$ in rats, a drastic increase in AMPK $\alpha$ expression was observed. Of note, the elevation of AMPK $\alpha$ expression preceded the decrease in Raptor and activation of ULK1, suggesting that an activated AMPK $\alpha$ facilitates inactivation of mTORC1, promotes the dissociation of the ULK1 compound along with the occurrence of autophagy.

Taken together, these results support the hypothesis that AMPK-mTOR-ULK1-mediated autophagy plays a role in the adaptive response protecting against $\mathrm{CCl}_{4}$-induced liver damage by inhibiting p21 (Fig. 5). Our findings pro- 


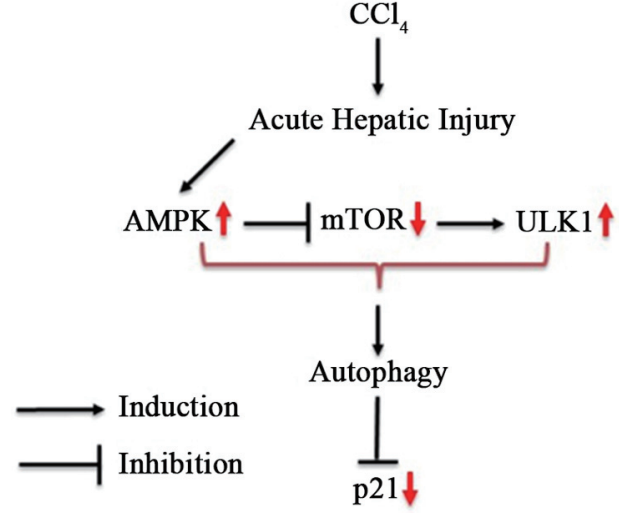

Fig. 5. Schematic of the association between autophagy and carbon tetrachloride $\left(\mathrm{CCl}_{4}\right)$-induced acute hepatic failure (AHF). $\mathrm{CCl}_{4}$-induced AHF causes exhaustion of hepatic energy, which subsequently activates AMPK $\alpha$, facilitates the inactivation of mTORC1, promotes the dissociation of the ULK1 compound and initiates the occurrence of autophagy. Autophagy can serve an important protective function against hepatic injury by inhibiting $\mathrm{p} 21$.

vide evidence for understanding the mechanism of $\mathrm{CCl}_{4} \mathrm{ac}$ tion in AHF.

Disclosure of Potential Conflicts of Interest: The authors declare that there are no conflicts of interest associated with this manuscript.

Acknowledgments: This study was financially supported by the Natural Science Foundation of China (31572270), the Key Scientific Research Projects of Henan Higher Education (19A180019, 18A180019), the National Fostering Science Foundation Project of Henan Normal University (2016PL21) and the Postdoctoral Research Grant in Henan Province (001803040).

\section{References}

1. Khan RA, Khan MR, and Sahreen S. $\mathrm{CCl}_{4}$-induced hepatotoxicity: protective effect of rutin on p53, CYP2E1 and the antioxidative status in rat. BMC Complement Altern Med. 12: 178. 2012.

2. Weber LW, Boll M, and Stampfl A. Hepatotoxicity and mechanism of action of haloalkanes: carbon tetrachloride as a toxicological model. Crit Rev Toxicol. 33: 105-136. 2003.

3. Rivera H, Shibayama M, Tsutsumi V, Perez-Alvarez V, and Muriel P. Resveratrol and trimethylated resveratrol protect from acute liver damage induced by $\mathrm{CCl}_{4}$ in the rat. J Appl Toxicol. 28: 147-155. 2008.

4. Ohyama T, Sato K, Kishimoto K, Yamazaki Y, Horiguchi N, Ichikawa T, Kakizaki S, Takagi H, Izumi T, and Mori M. Azelnidipine is a calcium blocker that attenuates liver fibrosis and may increase antioxidant defence. Br J Pharmacol. 165(4b): 1173-1187. 2012.

5. Toshima T, Shirabe K, Fukuhara T, Ikegami T, Yoshizumi
T, Soejima Y, Ikeda T, Okano S, and Maehara Y. Suppression of autophagy during liver regeneration impairs energy charge and hepatocyte senescence in mice. Hepatology. 60: 290-300. 2014.

6. Savini M, and Wang MC. Does autophagy promote longevity? It depends. Cell. 177: 221-222. 2019.

7. Mizushima N, Yoshimori T, and Levine B. Methods in mammalian autophagy research. Cell. 140: 313-326. 2010.

8. Wang XY, Yang H, Wang MG, Yang DB, Wang ZY, and Wang L. Trehalose protects against cadmium-induced cytotoxicity in primary rat proximal tubular cells via inhibiting apoptosis and restoring autophagic flux. Cell Death Dis. 8: e3099. 2017.

9. Yan S, Huda N, Khambu B, and Yin XM. Relevance of autophagy to fatty liver diseases and potential therapeutic applications. Amino Acids. 49: 1965-1979. 2017.

10. Codogno P, and Meijer AJ. Autophagy in the liver. J Hepatol. 59: 389-391. 2013.

11. Czaja MJ, Ding WX, Donohue TM Jr, Friedman SL, Kim JS, Komatsu M, Lemasters JJ, Lemoine A, Lin JD, Ou JH, Perlmutter DH, Randall G, Ray RB, Tsung A, and Yin XM. Functions of autophagy in normal and diseased liver. Autophagy. 9: 1131-1158. 2013.

12. Gual P, Gilgenkrantz H, and Lotersztajn S. Autophagy in chronic liver diseases: the two faces of Janus. Am J Physiol Cell Physiol. 312: C263-C273. 2017.

13. Wang G, Cheng L, Chen M, Zhao C, Gao M, Huang T, Chu $\mathrm{P}$, and $\mathrm{Xu} \mathrm{C}$. Comparative analysis of expression profiles of reg signaling pathways-related genes between AHF and HCC. Biochem Genet. 57: 382-402. 2019.

14. Mauthe M, Orhon I, Rocchi C, Zhou X, Luhr M, Hijlkema KJ, Coppes RP, Engedal N, Mari M, and Reggiori F. Chloroquine inhibits autophagic flux by decreasing autophagosome-lysosome fusion. Autophagy. 14: 1435-1455. 2018.

15. Mihaylova MM, and Shaw RJ. The AMPK signalling pathway coordinates cell growth, autophagy and metabolism. Nat Cell Biol. 13: 1016-1023. 2011.

16. Hernández-Gea V, Ghiassi-Nejad Z, Rozenfeld R, Gordon R, Fiel MI, Yue Z, Czaja MJ, and Friedman SL. Autophagy releases lipid that promotes fibrogenesis by activated hepatic stellate cells in mice and in human tissues. Gastroenterology. 142: 938-946. 2012.

17. He W, Wang B, Yang J, Zhuang Y, Wang L, Huang X, and Chen J. Chloroquine improved carbon tetrachloride-induced liver fibrosis through its inhibition of the activation of hepatic stellate cells: role of autophagy. Biol Pharm Bull. 37: 1505-1509. 2014.

18. Puls F, Goldschmidt I, Bantel H, Agne C, Bröcker V, Dämmrich M, Lehmann U, Berrang J, Pfister ED, Kreipe $\mathrm{HH}$, and Baumann U. Autophagy-enhancing drug carbamazepine diminishes hepatocellular death in fibrinogen storage disease. J Hepatol. 59: 626-630. 2013.

19. Jung J, Choi JH, Lee Y, Park JW, Oh IH, Hwang SG, Kim KS, and Kim GJ. Human placenta-derived mesenchymal stem cells promote hepatic regeneration in $\mathrm{CCl}_{4}$-injured rat liver model via increased autophagic mechanism. Stem Cells. 31: 1584-1596. 2013.

20. Kheloufi M, Boulanger CM, Durand F, and Rautou PE. Liver autophagy in anorexia nervosa and acute liver injury. BioMed Res Int. 2014: 701064. 2014.

21. Abbas T, and Dutta A. p21 in cancer: intricate networks and multiple activities. Nat Rev Cancer. 9: 400-414. 2009. 
22. Macip S, Igarashi M, Fang L, Chen A, Pan ZQ, Lee SW, and Aaronson SA. Inhibition of p21-mediated ROS accumulation can rescue p21-induced senescence. EMBO J. 21: 2180-2188. 2002.

23. Zou J, Ma Q, Sun R, Cai J, Liao H, Xu L, Xia J, Huang G, Yao L, Cai Y, Zhong X, and Guo X. Dihydroartemisinin inhibits HepG2.2.15 proliferation by inducing cellular senescence and autophagy. BMB Rep. 52: 520-524. 2019.

24. Manu KA, Cao PHA, Chai TF, Casey PJ, and Wang M. p21cip1/waf1 coordinate autophagy, proliferation and apoptosis in response to metabolic stress. Cancers (Basel). 11: e1112. 2019.

25. Gammoh N, and Wilkinson S. Autophagy in cancer biology and therapy. Front Biol. 9: 35-50. 2014.

26. Lesmana R, Sinha RA, Singh BK, Zhou J, Ohba K, Wu Y, Yau WW, Bay BH, and Yen PM. Thyroid hormone stimulation of autophagy is essential for mitochondrial biogenesis and activity in skeletal muscle. Endocrinology. 157: 23-38. 2016.

27. Jin Y, Bai Y, Ni H, Qiang L, Ye L, Shan Y, and Zhou M. Activation of autophagy through calcium-dependent AMPK/ mTOR and PKC $\theta$ pathway causes activation of rat hepatic stellate cells under hypoxic stress. FEBS Lett. 590: 672 682. 2016.

28. Padrissa-Altés S, Zaouali MA, Bartrons R, and RosellóCatafau J. Ubiquitin-proteasome system inhibitors and AMPK regulation in hepatic cold ischaemia and reperfusion injury: possible mechanisms. Clin Sci (Lond). 123: 93-98. 2012.

29. Zeng TS, Liu FM, Zhou J, Pan SX, Xia WF, and Chen LL. Depletion of Kupffer cells attenuates systemic insulin resistance, inflammation and improves liver autophagy in highfat diet fed mice. Endocr J. 62: 615-626. 2015. 